\title{
Revisiting Sebaceous Adenoma: Case Report and Discussion
}

\author{
Nishita Maganty ${ }^{1}$, Anngela Adams ${ }^{1}$, Matthew Muenster ${ }^{2}$, Kimberly Jared $^{3}$, Burrell Wolk ${ }^{3}$ and Neil \\ Fernandes $^{1,3^{*}}$
}

${ }^{1}$ University of Arizona College of Medicine-Phoenix, USA

${ }^{2}$ Miraca Life Sciences, USA

${ }^{3}$ Skin and Cancer Center of Arizona, USA

\begin{abstract}
Sebaceous adenomas are rare adnexal tumors that are associated with Muir-Torre syndrome. Although sebaceous adenomas are considered benign entities, this is controversial. It has been suggested that sebaceous adenomas are actually low-grade sebaceous carcinomas. In this report, we defend the view that sebaceous adenomas are in fact lowgrade sebaceous carcinomas by presenting a lesion with a clinical presentation typical for sebaceous carcinoma with histopathology characteristic of sebaceous adenoma.
\end{abstract}

\section{Keywords}

Sebaceous adenoma, Sebaceous carcinoma

\section{Introduction}

Sebaceous adenomas are benign, multilobar tumors with sebaceous differentiation [1,2]. The lobules extend into the reticular dermis, and may vary in both shape and size [1]. They can present as solitary or multiple lesions and are often yellow papules or nodules less than $0.5 \mathrm{~cm}$ in diameter [2,3]. Seventy percent are found on the head, with the most common site being the nose and cheek area, while $30 \%$ are found on the trunk, neck, and legs [3]. Occasionally, sebaceous adenomas may occur intraorally [3-5]. In the past, it has been suggested that there is a greater incidence in males [3]; however, recent literature indicates sebaceous adenomas affect both sexes equally [1]. Also, they are found more frequently in the elderly [6].

Sebaceous adenomas are the most common sebaceous tumors associated with Muir-Torre syndrome (MTS) [7], occurring in $68 \%$ of MTS patients [2]. MTS is an autosomaldominant genodermatosis characterized by mutations in DNA mismatch repair (MMR) enzymes [8,9]. Genes that encode DNA MMR enzymes include: MLH1, MSH2, MSH3, MLH3, MSH6, PMS1, and PMS2 [9]. These DNA MMR enzymes correct detected mismatched nucleotide bases [9]. The inability to correct mismatched nucleotide bases results in the accumulation of errors in microsatellite areas of DNA, leading to microsatellite instability [9]. This microsatellite instability predisposes MTS patients to sebaceous tumors and internal malignancies, including colorectal and genitourinary neoplasms [8]. In these patients, the sebaceous tumors occur most frequently on the trunk and may be the first clinical sign of MTS $[9,10]$.
Surgical excision is often utilized as treatment for sebaceous adenomas due to risk of recurrence [11]. In addition, although sebaceous adenoma is considered a benign entity, this is controversial. It has been suggested that sebaceous adenoma is a superficial or low-grade variant of sebaceous carcinoma [12-14]. A. Bernard Ackerman, respected dermatologist and dermatopathologist, first proposed the idea that sebaceous adenomas were in fact sebaceous carcinomas due to the variable presence of mitotic figures, pleomorphism, and crowded nuclei in these lesions [14]. Misago and Narisawa suggested that benign sebaceous neoplasms in MTS may be well-differentiated sebaceous carcinomas with low-grade malignancy or have potential for malignant transformation [15]. Herein we describe the case of a patient with a clinical lesion concerning for sebaceous carcinoma, but with a histopathological picture classic for sebaceous adenoma.

\section{Case Report}

A 68-year-old man presented to the dermatology clinic

* Corresponding author: Neil F Fernandes, MD, Skin and Cancer Center of Arizona; University of Arizona College of Medicine-Phoenix, 725 S Dobson Rd Suite 200, Chandler, AZ 85224, USA, Tel: (480)-899-7546

Accepted: May 08, 2019

Published online: May 10, 2019

Citation: Maganty N, Adams A, Muenster M, et al. (2018) Revisiting Sebaceous Adenoma: Case Report and Discussion. Dermatol Arch 3(1):77-79 


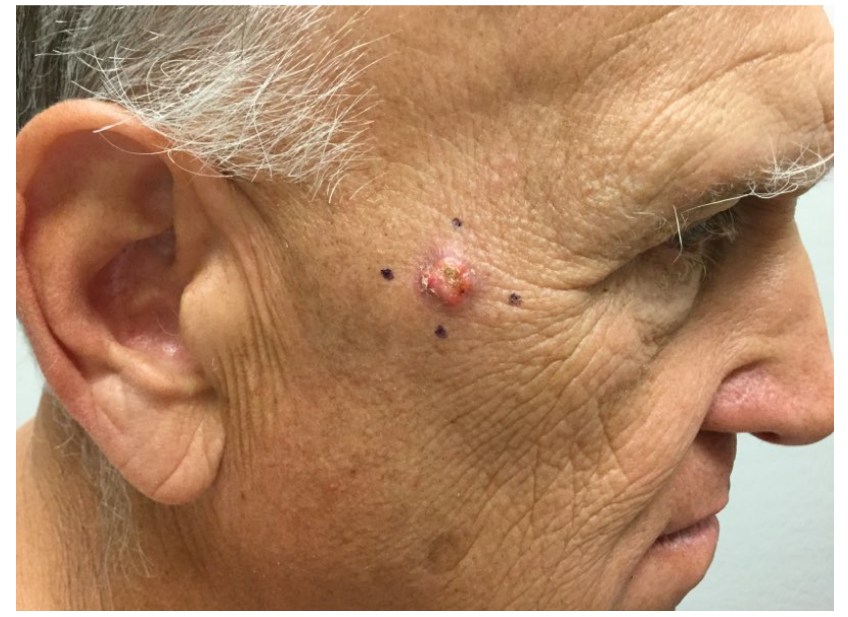

Figure 1: A clinical photograph of a bleeding lesion on the right central zygoma of the patient.

3

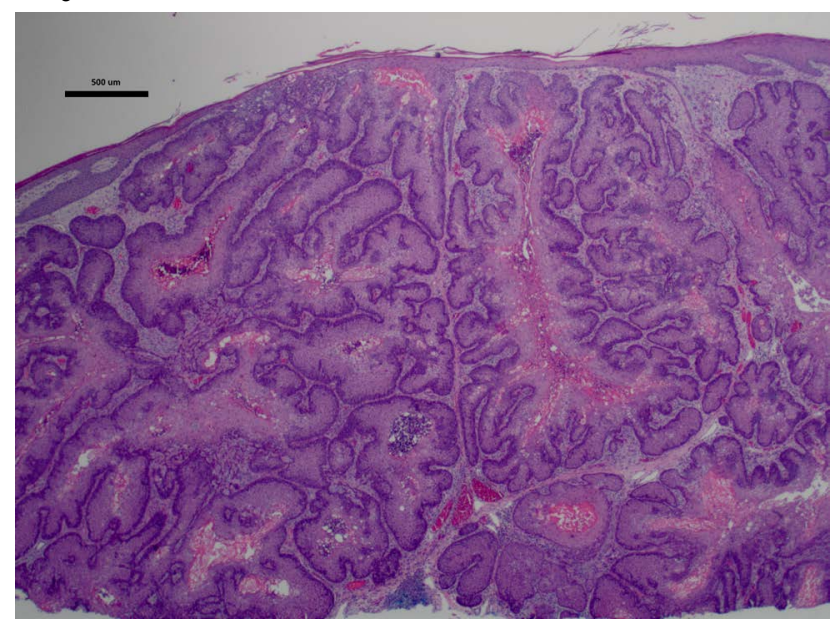

Figure 2: Low magnification image showing multiple circumscribed sebaceous lobules with a peripheral basaloid layer.

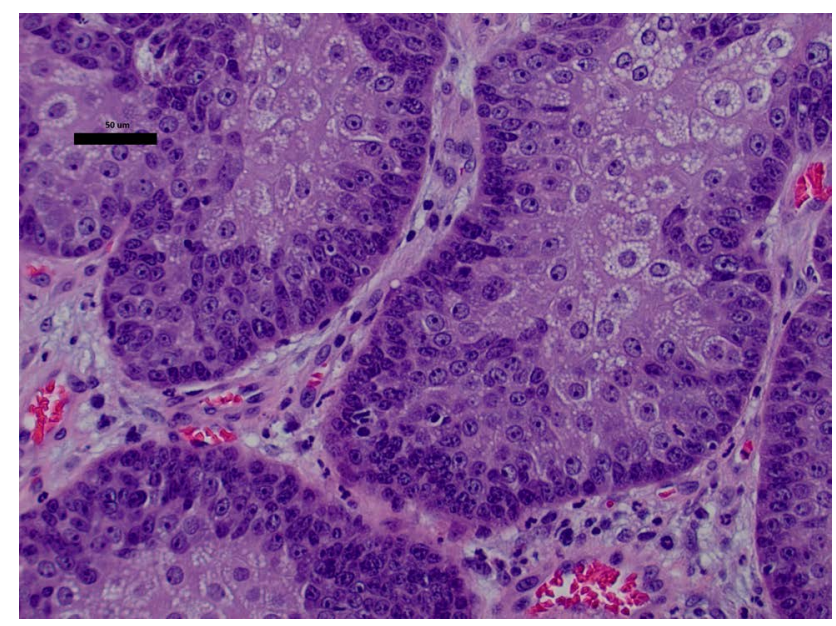

Figure 3: High magnification shows blue islands with numerous sebaceous cells. with a solitary enlarging, bleeding lesion on his right central zygoma (Figure 1). The patient had no significant past medical or surgical history. The lesion was present for 6 months. Physical examination revealed a $1 \mathrm{~cm}$ reddish, orange and yellow nodule with focal crusting (Figure 1). The differential diagnosis included squamous cell carcinoma and basal cell carcinoma. A shave biopsy was performed, which showed multiple circumscribed sebaceous lobules with a peripheral basaloid layer at scanning magnification (Figure 2). High power microscopy showed blue islands with numerous sebaceous cells (Figure 3). There were few scattered mitoses, approximately 2.5 per square millimeter. There was no obvious pleomorphism, hyperchromasia or cellular necrosis. Based on these histopathological findings, a diagnosis of sebaceous adenoma was given. The patient was referred for complete excision of the lesion given the potential for local recurrence. The excision of the lesion demonstrated scar from the prior biopsy without residual sebaceous neoplasm. The patient's sebaceous adenoma was determined to be sporadic in origin due to no family history of MTS, no personal history of cancer, and a normal colonoscopy performed prior to the biopsy of the lesion.

\section{Discussion}

Clinically, sebaceous adenomas often present as wellcircumscribed, smooth, yellow papules less than $0.5 \mathrm{~cm}$ in diameter [2]. They are most often found on the forehead and cheeks although they can be found beyond the head and neck area $[7,16]$. Sebaceous carcinomas are potentially fatal malignant adenocarcinomas that most commonly are located on the head and neck as well [17]. The clinical presentation is varied; however, they usually present as erythematous to yellow, firm nodules with hemorrhagic crust [17-19]. Extraocular sebaceous carcinomas range in size from $6 \mathrm{~mm}$ to $8 \mathrm{~cm}$ [20]. The variable clinical presentation for sebaceous carcinomas often leads to difficulty and delay in diagnosis [21]. Clinically, the difference between sebaceous adenomas and carcinomas may be subtle. This is supported in the presented case, as the $1 \mathrm{~cm}$ reddish, orange and yellow nodule with focal crusting did not have histopathologic features of sebaceous carcinoma. Conversely, there have been reports of sebaceous carcinomas presenting clinically as fairly well-circumscribed, symmetric, smooth lesions [22]. This demonstrates the overlapping clinical presentation that can be seen between sebaceous adenomas and sebaceous carcinomas.

Histopathologically, sebaceous adenomas are multilobar lesions with peripheral basaloid cells expanded beyond two layers [1]. They often have central mature sebocytes that are larger with eosinophilic, bubbly cytoplasm [12]. Furthermore, they are well-demarcated, without widespread pleomorphism, hyperchromasia, and cellular necrosis [23]. Sebaceous carcinomas are poorly circumscribed, with pleomorphic tumor cells, necrosis, hyperchromatism, and high mitotic activity [16]. Ackerman noted sebaceous adenomas may have overlapping histopathological features with sebaceous carcinoma such as occasional mitoses, pleomorphism, and nuclear crowding [14].

Therefore, cutaneous adnexal tumors with sebaceous dif- 
ferentiation may be difficult to classify [24]. As seen in the presented patient, the clinical presentation was more concerning for sebaceous carcinoma versus sebaceous adenoma. However, on histopathology the lesion had textbook features of sebaceous adenoma and lacked the atypia and disorganization seen with sebaceous carcinoma. This further suggests that there are similar features between sebaceous carcinomas and sebaceous adenomas. This diagnostic dilemma is frequently encountered, as sebaceous tumors are increasingly being classified as atypical sebaceous neoplasms [25].

This case supports Ackerman's suggestion that sebaceous adenomas are representative of low-grade sebaceous carcinomas [14]. Given that sebaceous adenomas are normally excised, it is difficult to say whether they would metastasize if left untreated. We cannot advocate for excision of all lesions that clinically present as sebaceous adenomas. However, excision should be seriously consideration given the risk of local recurrence as well as the evidence that sebaceous adenomas may actually be low-grade sebaceous carcinomas.

\section{References}

1. Marques-da-Costa J, Campos-do-Carmo G, Ormiga $P$, et al (2015) Sebaceous adenoma: Clinics, dermatoscopy, and histopathology. Int J Dermatol 54: 200-202.

2. Eisen DB, Michael DJ (2009) Sebaceous lesions and their associated syndromes: Part I. J Am Acad Dermatol 61: 549-560.

3. Prioleau PG, Santa Cruz DJ (1984) Sebaceous gland neoplasia. J Cutan Pathol 11: 396-414.

4. Izutsu T, Kumamoto H, Kimizuka S, et al. (2003) Sebaceous adenoma in the retromolar region: Report of a case with a review of the English literature. Int J Oral Maxillofac Surg 32: 423-426.

5. Orlian Al, Salman L, Reddi T, et al. (1987) Sebaceous adenoma of the oral mucosa. J Oral Med 42: 38-39.

6. Meer S (2010) Rook's textbook of dermatology. Blackwell Publishing, UK.

7. Flux K (2017) Sebaceous Neoplasms. Surg Pathol Clin 10: 367382.

8. Cohen PR, Kohn SR, Kurzrock R (1991) Association of sebaceous gland tumors and internal malignancy: The muir-torre syndrome. Am J Med 90: 606-613.

9. Singh RS, Grayson W, Redston M, et al. (2008) Site and tumor type predicts DNA mismatch repair status in cutaneous sebaceous neoplasia. Am J Surg Pathol 32: 936-942.

10. Moscarella E, Argenziano G, Longo C, et al. (2013) Clinical, dermoscopic and reflectance confocal microscopy features of sebaceous neoplasms in Muir-Torre syndrome. J Eur Acad Dermatol Venereol 27: 699-705.

11. Dhingra KK, Saroha V, Gupta P, et al. (2009) Demodex-associated dermatologic conditions--A coincidence or an etiological correlate. Review with a report of a rare case of sebaceous adenoma. Pathol Res Pract 205: 423-426.

12. Lazar AJ, Lyle S, Calonje E (2007) Sebaceous neoplasia and TorreMuir syndrome. Curr Diagn Pathol 13: 301-319.

13. Hookerman BJ (2013) Dermatopathology: An abridged compendium of words. A discussion of them and opinions about them. Introduction and part 1. Dermatol Pract Concept 3: 13-40.

14. Ackerman A, Nussen S, Tan M (1998) Histopathologic Diagnosis of Neoplasms with Sebaceous Differentiation. Ardor Scribendi, UK.

15. Misago N, Narisawa $Y(2000)$ Sebaceous neoplasms in MuirTorre syndrome. Am J Dermatopathol 22: 155-161.

16. Danialan R, Mutyambizi K, Aung P, et al. (2015) Challenges in the diagnosis of cutaneous adnexal tumours. J Clin Pathol 68: 992-1002.

17. Moon TC, Cassler NM, Lackey JN (2015) Sebaceous carcinoma on the abdomen in an African-American male patient. Indian Dermatol Online J 6: 27-29.

18. Dasgupta T, Wilson LD, Yu JB (2009) A retrospective review of 1349 cases of sebaceous carcinoma. Cancer 115: 158-165.

19. Lucares DO, Bernardes Filho F, Vega H, et al. (2013) Inguinal ulcerated sebaceous carcinoma: An unusual presentation. An Bras Dermatol 88: 48-51.

20. Bailet JW, Zimmerman MC, Arnstein DP, et al. (1992) Sebaceous carcinoma of the head and neck. Case report and literature review. Arch Otolaryngol Head Neck Surg 118: 1245-1249.

21. Brady KL, Hurst EA (2017) Sebaceous carcinoma treated with mohs micrographic surgery. Dermatol Surg 43: 281-286.

22. Kazakov DV, Kutzner H, Spagnolo DV, et al. (2009) Discordant architectural and cytological features in cutaneous sebaceous neoplasms--a classification dilemma: report of 5 cases. Am J Dermatopathol 31: 31-36.

23. Crowson AN, Magro CM, Mihm MC (2006) Malignant adnexal neoplasms. Mod Pathol 19: S93-S126.

24. Elston DM (2016) Sebaceous Adenoma. Medscape.

25. Mojtahed A, Schrijver I, Ford JM, et al. (2011) A two-antibody mismatch repair protein immunohistochemistry screening approach for colorectal carcinomas, skin sebaceous tumors, and gynecologic tract carcinomas. Mod Pathol 24: 1004-1014.

DOI: $10.36959 / 661 / 305$

Copyright: (c) 2019 Maganty N, et al. This is an open-access article distributed under the terms of the Creative Commons Attribution License, which permits unrestricted use, distribution, and reproduction in any medium, provided the original author and source are credited. 